\title{
Electrical transport within polymeric amorphous carbon thin films and the effects of ion implantation
}

\author{
R. U. A. Khan ${ }^{\mathrm{a})}$ \\ Blackett Laboratory, Imperial College London, Prince Consort Road, London SW7 2AZ, United Kingdom \\ S. R. P. Silva \\ School of Electronics and Physical Sciences, University of Surrey, Guildford, Surrey GU2 7XH, \\ United Kingdom
}

(Received 11 April 2003; accepted 26 June 2003)

\begin{abstract}
Here, we will discuss the electrical transport processes that occur within thin films of polymeric amorphous carbon, which possess a high resistivity of $10^{15} \Omega \mathrm{cm}$, a Tauc band gap of $2.6 \mathrm{eV}$, and a low defect density of $10^{17}$ spins $\mathrm{cm}^{-3}$. Using current versus voltage measurements, we have shown that the current is space-charge-limited, with a mobility value of typically $10^{-10} \mathrm{~cm}^{2} \mathrm{~V}^{-1} \mathrm{~s}^{-1}$. The implantation of boron ions at doses below $6 \times 10^{14} \mathrm{~cm}^{-2}$ results in an increase in mobility of more than one order of magnitude. Above this dose, the conductivity increases by five orders of magnitude, and the transport mechanism changes to a Frenkel-Poole type conduction process. At an intermediate dose of $2 \times 10^{15}$ ions $^{-2}$, the current versus voltage characteristic exhibits strong hysteresis. The observed hysteresis effects could be removed from one polarity by implanting only through the first half of the film. The hysteresis is likely to be due to the trapping of holes at one or both of the interfaces, resulting in the modification of the space-charge within the polymeric amorphous carbon film. (C) 2003 American Institute of Physics.
\end{abstract}

[DOI: $10.1063 / 1.1602953$ ]

\section{INTRODUCTION}

For several years, there has been much interest in the material known as hydrogenated amorphous carbon $(a-\mathrm{C}: \mathrm{H})$, both due to its outstanding mechanical properties, and also its potential in electronic applications due to its tunable optical band gap. ${ }^{1}$ Various types of this material have been proposed as viable alternatives to amorphous silicon for applications such as solar cells and thin-film transistors. Furthermore, thin films of amorphous carbon have been shown to exhibit electron field emission, ${ }^{2}$ so these films may be a viable emissive layer for the next generation of flat-panel displays. However, its electronic properties are limited by the fact that, in most forms of the material, the transport at high electric fields $\left(>10^{5} \mathrm{~V} \mathrm{~cm}^{-1}\right)$ is normally governed by the field assisted excitation of carriers which are trapped within Coulombic wells, which is known as Frenkel-Poole type conduction. ${ }^{3,4}$ This type of mechanism occurs in materials which possess a very high defect density, such as in various forms of $a-\mathrm{C}: \mathrm{H}$ where values of approximately $10^{20}$ paramagnetic spins $\mathrm{cm}^{-3}$ (Ref. 5) have been observed. Other studies have demonstrated the usability of amorphous carbon for metal-insulator-metal switches ${ }^{6,7}$ and shown the formation of a Schottky barrier between amorphous carbon and some metals. ${ }^{8}$

Polymeric amorphous carbon (PAC) possesses a significantly lower defect density of approximately $10^{17}$ spins $\mathrm{cm}^{-3}$, and therefore is more promising as an electronic material, as well as also being a viable field-emitting

a) Author to whom correspondence should be addressed; electronic mail: riz.khan@ic.ac.uk material. ${ }^{9,10}$ We have demonstrated that the material possesses a high resistivity in its as-deposited state, but this may be controllably reduced by ion implantation, which results in a partial delocalization of the film at low ion doses, ${ }^{11}$ although graphitization occurs above an ion dose of 6 $\times 10^{14} \mathrm{~cm}^{-2}$, which manifests itself as a decrease in optical band gap. Previous to this study, the ion implantation of amorphous carbon has been studied, but only on materials possessing a high initial defect density, and extensive $s p^{2}$ restructuring $^{12-14}$ was observed.

In this study, we will investigate the transport properties of PAC in its as-deposited state, which have been measured by fabricating metal-semiconductor-metal diodes. The effects of boron ion-implantation will also be observed in order to ascertain the optical and electronic processes that are involved.

\section{EXPERIMENT}

Our films were deposited on the earthed substrate of an rf-plasma enhanced chemical vapor deposition reactor at room temperature, which produced polymeric amorphous carbon films with a hydrogen content of $\sim 55$ at. $\%$, as measured using elastic recoil detection analysis, and a low paramagnetic defect density of $\sim 10^{17} \mathrm{~cm}^{-3}$ (Ref. 9) as measured using electron paramagnetic resonance spectroscopy. The films were grown onto metallic chromium nitride substrates. Some samples were then implanted with boron ions at energies of 20 and $26 \mathrm{keV}$, with projected ranges of 60 and 76 $\mathrm{nm}$. Metal-semiconductor-metal (MSM) structures were fabricated by evaporating gold top contacts upon the films, which possessed a diameter of between 0.25 and $1 \mathrm{~mm}$, and 
a thickness of approximately $200 \mathrm{~nm}$. Devices were fabricated using different contact materials ( $\mathrm{Ti}, \mathrm{Cr}, \mathrm{Al})$ and it was found that the $J-V$ characteristics were independent of the electrode.

We then measured the electrical characteristics of these devices by performing current versus voltage measurements using a Keithley 487 picoammeter/voltage source. Measurements were performed parametric in temperature over a range of $300-550 \mathrm{~K}$. Before these measurements were performed, the sample was annealed at $300^{\circ} \mathrm{C}$ for $5 \mathrm{~min}$ in a vacuum better than $10^{-5} \mathrm{mbar}$, in order to ensure temperature stability. Resistivity values were derived from the slope of the $J$ versus $E$ characteristic at electric fields of $<2$ $\times 10^{5} \mathrm{~V} \mathrm{~cm}^{-1}$. Films were also deposited onto Corning 7059 glass substrates and optical absorption measurements were performed on these samples, in order to measure the band gap of the material. The thicknesses of the films were derived using ellipsometry, by using a $632.5 \mathrm{~nm} \mathrm{He} / \mathrm{Ne}$ source, and also confirmed using profilometry.

\section{RESULTS}

The current density $(J)$ versus electric field $(E)$ of a typical $100 \mathrm{~nm}$ thick PAC film is shown in Fig. 1(a) as a linear plot. This is followed by the same data shown as a logarithmic $J$ versus $E$ plot, and as $\ln J$ versus $E^{1 / 2}$ for the positive half cycle only, in Figs. 1(b) and 1(c). It can seen from Fig. (a) that up to an electric field of $4 \times 10^{5} \mathrm{~V} \mathrm{~cm}^{-1}$ the current density scales approximately linearly with the electric field which allows low-field values of resistivity to be calculated. For this film the calculated resistivity is $2 \times 10^{15} \Omega \mathrm{cm}$, and comparable to some of the better insulators available for thin films. These films have been used as passivation layers for high voltage power devices due to this property. A linear $J / E$ characteristic at these electric fields suggests that electronic transport is occurring either by hopping around the Fermi level, conduction in the band tails, or conduction through extended states, ${ }^{15,16}$ rather than any of the mechanisms that apply in the high field, such as space-charge-limited current, ${ }^{17}$ Frenkel-Poole conduction, ${ }^{18}$ or the Schottky effect. ${ }^{19}$

It is shown in Fig. 1(b) that there is a large degree of hysteresis in the $J / E$ characteristic, as when the voltage is reduced from the maximum value, the measured current is lower than the current which was measured on the ramp-up. This is most likely to be an effect of the capacitance of the device, and the long time constant which is as a result of the high resistivity of the film.

Figure 1(c) plots $\ln J$ versus $E^{1 / 2}$, which is the conventional graph ${ }^{20}$ for the purpose of fitting to Frenkel-Poole or Schottky effect conduction mechanisms. This figure shows that an approximately straight-line region is obtained in the $E^{1 / 2}$ range from 400 to $700 \mathrm{~V}^{1 / 2} \mathrm{~cm}^{-1 / 2}$. Therefore, it is possible that either of these two mechanisms is applicable. The analysis of the slope will be performed in the Discussion.

Figure 2 shows the characteristics of films grown to different thicknesses in order to check whether conduction is contact or bulk limited. Should conduction be contact limited, $J$ should be the same irrespective of film thickness when
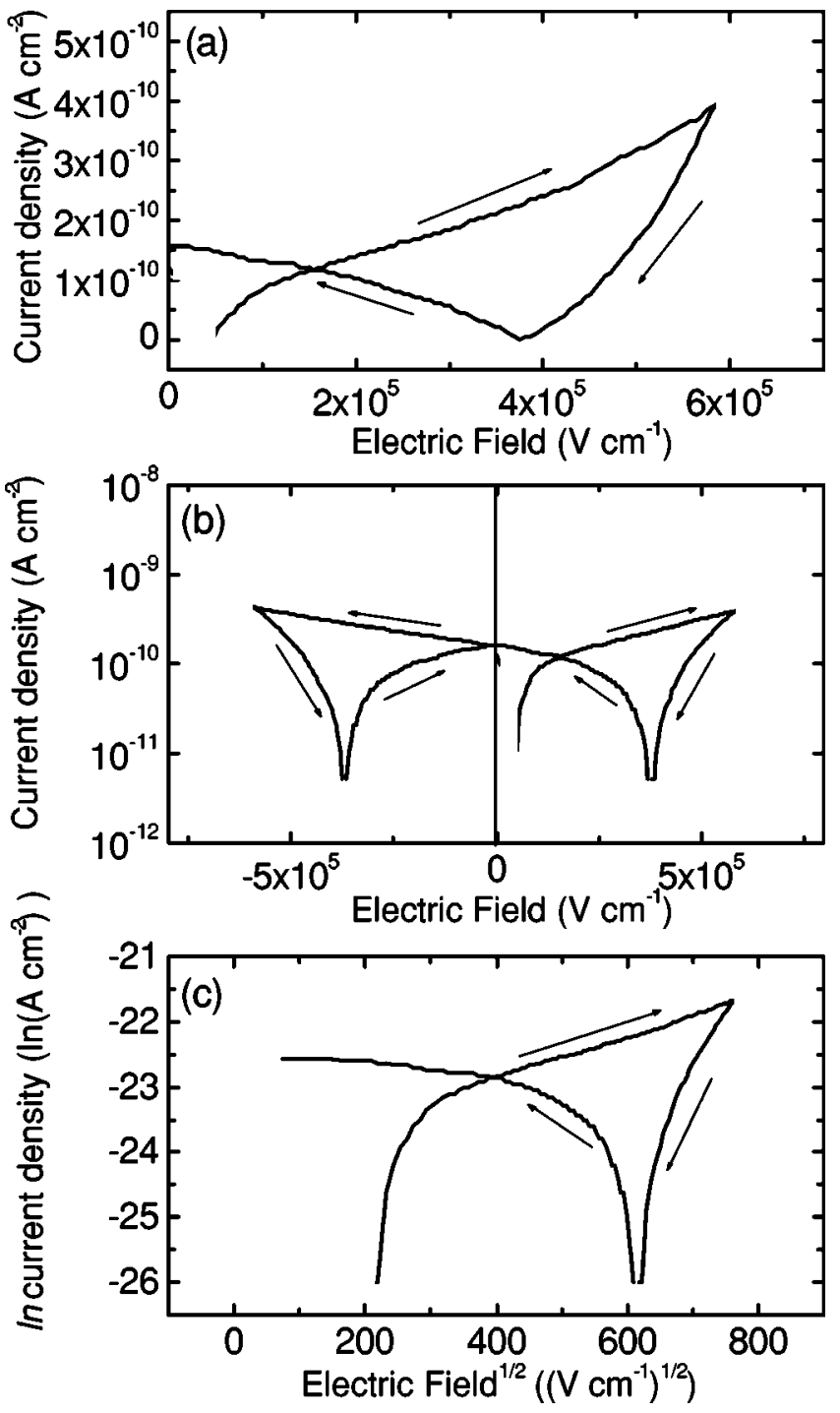

FIG. 1. Current vs voltage characteristics as-deposited PAC films: (a) linear $J$ versus $E$ plot; (b) logarithmic $J$ vs $E$ plot; and (c) $\ln J$ vs $E^{1 / 2}$ plot.

plotted against $V$, if the depletion region does not extend throughout the length of the film. ${ }^{21}$ It can be seen in Fig. 2(a) that the $J$ versus $V$ characteristics do not coincide for the three film thicknesses, which renders a contact-limited conduction process under these conditions unlikely. If either the conduction was contact limited but the depletion region extended throughout the device, or was governed by a bulklimited process such as hopping or Frenkel-Poole conduction, then the current would scale with the electric field over the device, so the $J$ versus $E$ characteristic would be the same for all film thicknesses. This is plotted in Fig. 2(b). It can be seen that once again the $J$ versus $E$ curves do not coincide for the three film thicknesses.

A common high-field conduction mechanism in MSM structures is space-charge-limited current (SCLC) where the dependence follows a parabolic relationship which varies according to the density and distribution of states within the band gap. Figure 2(c) shows the variation of $J / d$ against $V / d^{2}$ on $\log -\log$ axes for the three film thicknesses to investigate if SCLC is governing high-field conduction according to the equation shown in the Discussion section. It may be 

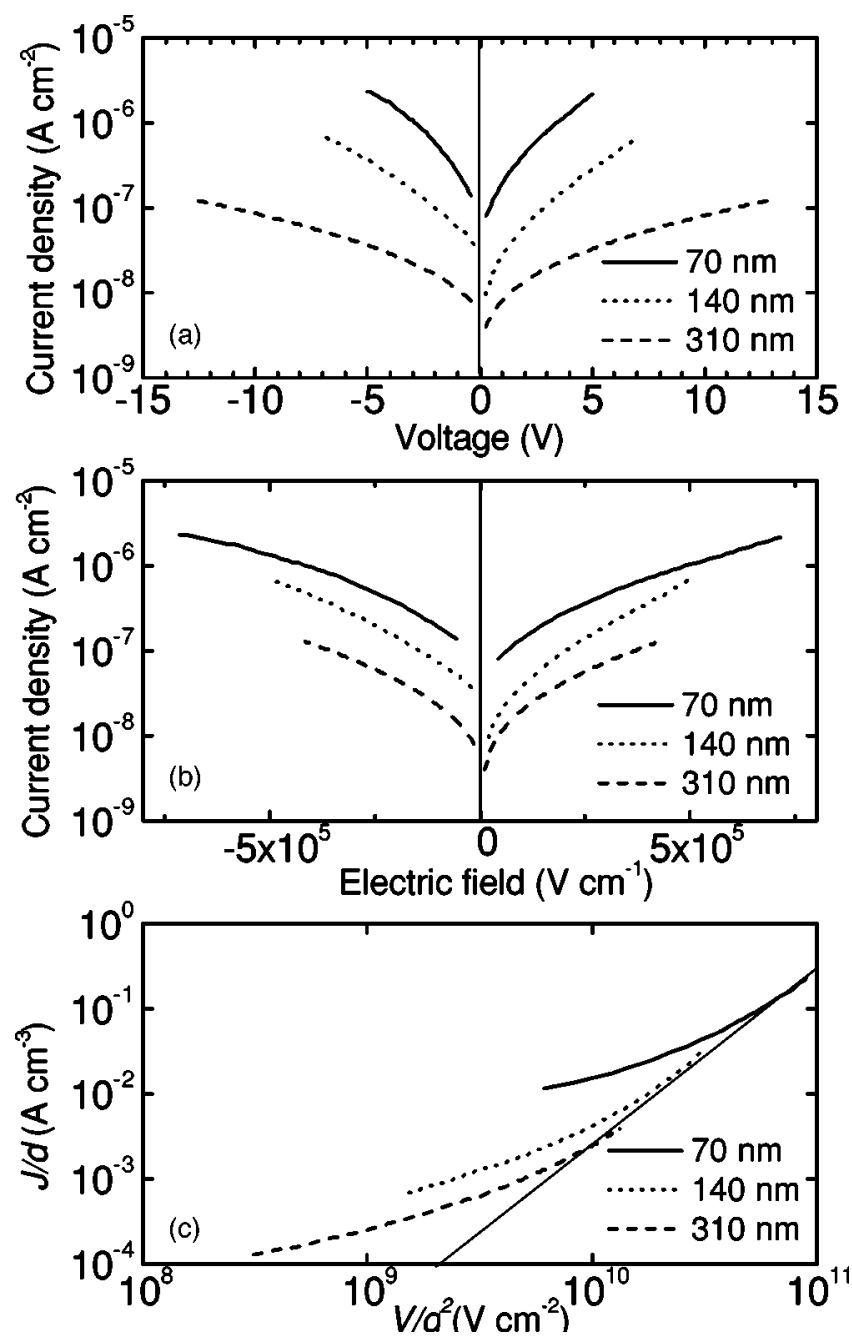

FIG. 2. Current vs voltage characteristics for as deposited PAC films of differing thicknesses: (a) $J$ vs $V$ plot; (b) $J$ vs $E$ plot; and (c) $J / d$ vs $V / d^{2}$ plot.

observed that although the curves for the three different film thicknesses do not coincide at low voltages, they approach a similar line and are coincident at higher voltages. A slope may be calculated from this line and is found to be 2.0. This suggests a parabolic dependence of current against voltage, i.e., $J \propto V^{2.0}$, and indicates SCLC at high fields only.

In order to observe whether conduction occurred via carriers excited into the extended states, into localized states in the band tail, or by hopping at the Fermi level (either thermally activated or variable-range hopping), $J$ versus $E$ measurements were performed as a function of temperature. ${ }^{15}$ Figure 3 shows the variation of $\ln$ (conductivity) against $-q / k T$ for a PAC film. It may be observed that at low $-q / k T$ values, corresponding to a measurement temperature of less than $100^{\circ} \mathrm{C}$, there is almost no variation of conductivity with temperature, which means that the activation energy in this range is close to zero. At higher temperatures $\left(>110^{\circ} \mathrm{C}\right)$ a straight-line dependence is observed for $\ln J$ versus $-q / k T$ plot which suggests a thermally activated process which fits the Arrhenius law. From the slope of the graph an activation energy may be determined and the value

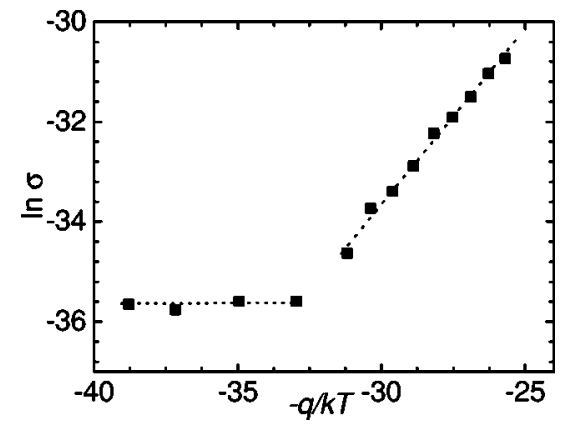

FIG. 3. In (conductivity) against $-q / k T$ for PAC, from which carrier activation energy values may be derived.

derived is $0.7 \mathrm{eV} \pm 0.05 \mathrm{eV}$. This compares with a Tauc optical band gap value of $2.6 \mathrm{eV}$.

We will now show the variation in these electrical properties as a function of boron ion dose. The $J$ versus $E$ characteristic of the PAC diodes over the range of boron ion doses is shown in Fig. 4. Apart from the curve at 2 $\times 10^{15} \mathrm{~cm}^{-2}$, the characteristics are symmetrical with the magnitude of the current increasing by many orders of magnitude as the dose increases. It is interesting to note that a large range in conductivity may be achieved over a narrow dose range, indicating that the predominant reordering and increase in conductivity occurs over this range. Furthermore, the film implanted at $2 \times 10^{15} \mathrm{~cm}^{-2}$ shows asymmetry which manifests itself as increased hysteresis in the negative sweep.

Figure 5 shows the $J$ versus $E$ characteristics for two films implanted at a dose of $2 \times 10^{15} \mathrm{~cm}^{-2}$ with as-deposited thicknesses of 120 and $190 \mathrm{~nm}$, respectively. Following implantation the thicknesses had reduced to 80 and $104 \mathrm{~nm}$. Upon applying a positive voltage to the top contact, the current increases and there is a pronounced hysteresis with a larger current on the return sweep to zero voltage. Also, it can be seen that in the positive sweep the $80 \mathrm{~nm}$ thick film is one order of magnitude higher in conductivity than the 104 $\mathrm{nm}$ thick film; however, the characteristics are similar with hysteresis resulting in a current enhancement. The negative sweep shows an even larger degree of hysteresis in the 80 $\mathrm{nm}$ thick film and no hysteresis in the $104 \mathrm{~nm}$ thick film.

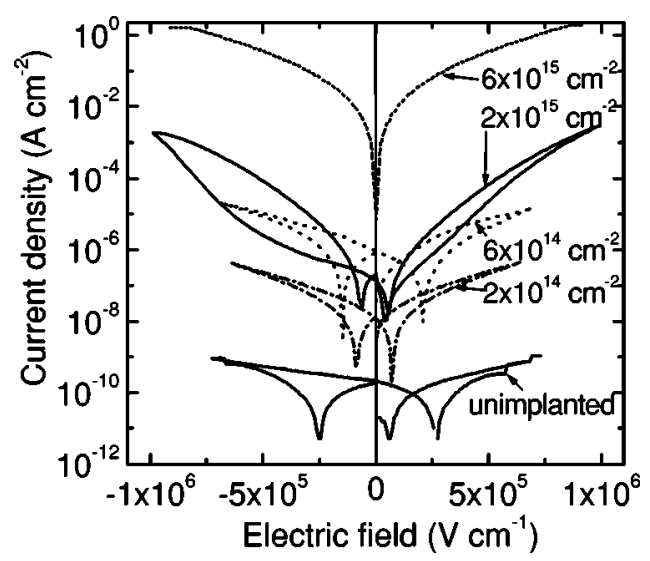

FIG. 4. Variation in the current density vs applied electric field characteristic as a function of boron ion dose. 


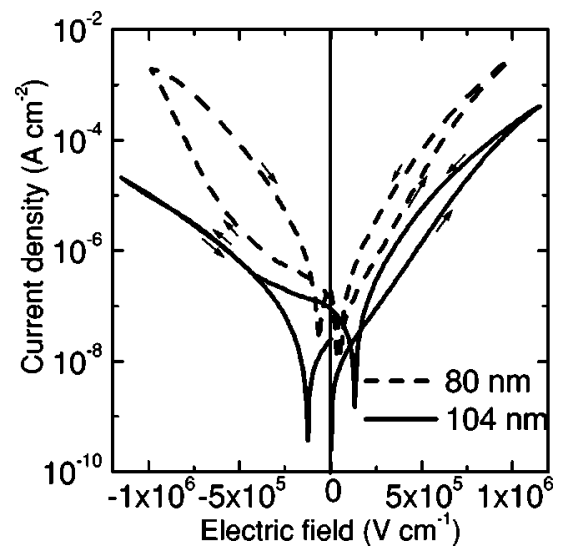

FIG. 5. $J$ vs $E$ characteristic for films implanted with $2 \times 10^{15} \mathrm{~cm}^{-2}$ boron ions, possessing thicknesses of 80 and $104 \mathrm{~nm}$.

Therefore, the $104 \mathrm{~nm}$ thick film shows hysteresis in one sweep only which results in rectification of approximately one order of magnitude.

It was thought that the behavior of trapped carriers might be influencing the current density of these films, as this type of hysteresis might be expected if carriers are trapped and decay with a long time constant. Therefore, current versus time measurements were performed at varying voltages for the sample implanted with boron ions at $2 \times 10^{15} \mathrm{~cm}^{-2}$. The resulting characteristics are shown in Fig. 6. It can be seen that up to an applied voltage of $3 \mathrm{~V}$, the current decays with time in a manner which might be expected if the device is considered as a parallel-plate capacitor. However, above the voltage of $3 \mathrm{~V}$ (which coincides with voltages above the switching electric field of the film) the current shows an increase with time. This is perhaps surprising as it is not commonly observed in most dielectric materials.

In order to supplement the electrical data, measurements were performed as a function of temperature up to $160^{\circ} \mathrm{C}$, which is shown in Fig. 7. It may be observed that between measurement temperatures of 23 to $80^{\circ} \mathrm{C}$, the current density decreases by approximately one order of magnitude; in other words, the film possesses a negative activation energy of $0.5 \pm 0.05 \mathrm{eV}$. A negative activation energy has seldom been reported in the literature for thin-film materials. At higher

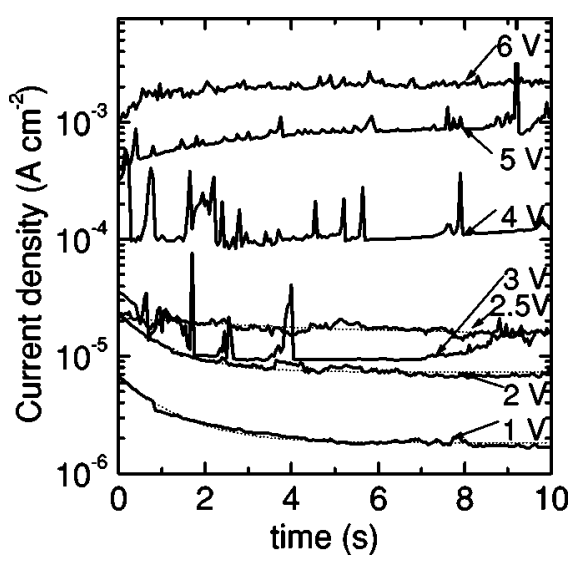

FIG. 6. Current density vs time characteristic for $2 \times 10^{15} \mathrm{~cm}^{-2} \mathrm{~B}^{+} \mathrm{im}-$ planted PAC film at varying voltages.

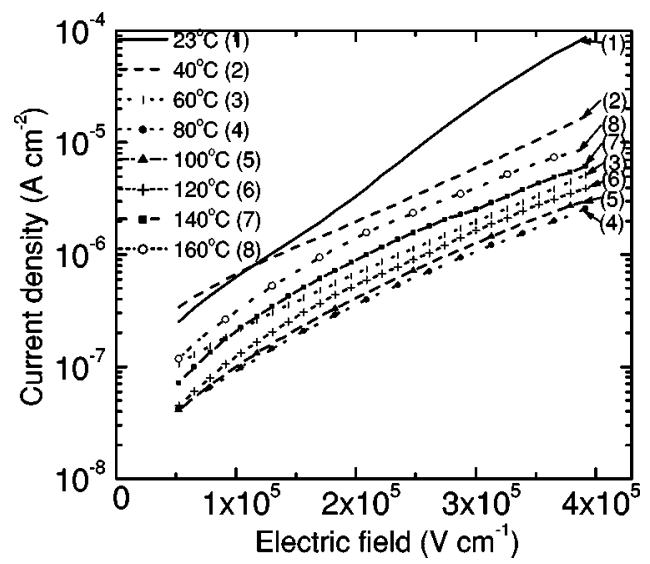

FIG. 7. Variation in positive voltage sweep of $J / E$ characteristic as a function of measurement temperature.

temperatures the activation energy becomes positive with a value of $0.32 \pm 0.03 \mathrm{eV}$. This coincides with a reduction in hysteresis up to the measurement temperature of $80^{\circ} \mathrm{C}$, which is not shown in the plot for the purposes of clarity. Above $80^{\circ} \mathrm{C}$ there is no longer any hysteresis in the $J / E$ characteristic.

\section{DISCUSSION}

It can be seen in Fig. 1 that the current rise with applied voltage is linear at low fields and that the characteristics show a large degree of hysteresis which suggests either a large contribution of device capacitance or trapping. It has been observed that as the time interval between measurements is increased between $0.5 \mathrm{~s}$ per $0.1 \mathrm{~V}$ measurement step to $2 \mathrm{~s}$ per measurement step, the degree of hysteresis is seen to decrease. This suggests that there is an initial increase in current as the voltage is increased which decays as the measurement time is increased, which would suggest a capacitive effect. Assuming $C=A \epsilon_{0} \epsilon_{r} / d$ and $R=\rho d / A$, the time constant $R C=\rho \epsilon_{0} \epsilon_{r}$. If $\epsilon_{r}=2.6$, i.e., the square of the refractive index, and $\rho=2 \times 10^{15} \Omega \mathrm{cm}$, then the calculated time constant is approximately $460 \mathrm{~s}$. Therefore, over the time of measurement performed here, a considerable residual voltage caused by the capacitance of the device $\left(V_{C}\right)$ exists over the device due to stepping up to the maximum voltage value even as the measurement voltage $\left(V_{M}\right)$ is then stepped back down to zero. Therefore, the overall voltage across the film is lower than the applied voltage due to the capacitance contribution, as $V_{\mathrm{TOT}}=V_{M}-V_{C}$, and this in turn is most likely to account for the hysteresis. In order to obtain an accurate analysis of the hysteresis we need to conduct extensive $C$ - $V$ analysis as a function of frequency. But, preliminary results give a flat response with voltage that indicates no change in the depletion region width with bias, and capacitance values close to those calculated using the above equation, for the three film thicknesses.

In order to ascertain whether high-field conduction may be governed by Frenkel-Poole or Schottky conduction, it is necessary to investigate Fig. 1(c). The Frenkel-Poole and Schottky equations are as follows, respectively: $:^{22,21}$ 


$$
J=E e \mu N_{c} \exp \left[\frac{E_{g}}{2 k T}\right] \exp [\beta \sqrt{E}]
$$

and

$$
J=A^{* *} T^{2} \exp \left[\frac{\Phi_{0}}{k T}+\frac{\beta}{2} \sqrt{E}\right],
$$

where

$$
\beta=\frac{1}{k T} \sqrt{\frac{e^{3}}{\pi \epsilon_{0} \epsilon_{r}}}
$$

and $A^{* *}$ is Richardson's constant, $k$ is Boltzmann's constant, $T$ is the absolute temperature, $e$ is $1.6 \times 10^{-19} C, \epsilon_{0}$ is the permittivity of free space, and $\epsilon_{r}$ is the relative permittivity. By applying these equations and ignoring for the time being the $E$ prefactor which would only effect the values in the low field, the derived value of permittivity is 110 , which is far higher than the expected value derived from the square of refractive index, which is approximately 2.6. Therefore, it is highly unlikely that the Frenkel-Poole effect governs the conduction process within these films. The Schottky effect is also unlikely as in this case a slope of $\beta / 2$ would yield a dielectric constant value of approximately 28 , which is also far higher than the expected value. Furthermore, the fact that the measurements are independent of the choice of electrode indicates that the characteristics are not contact-limited.

SCLC has been used in order to fit to the $J / E$ characteristics to obtain the density of states within the band gap. ${ }^{23,24}$ However, in the case of this material, the trap density is not as high as in the case of previously observed $a-\mathrm{C}: \mathrm{H}$ films and therefore a simpler analysis assuming either a trap-free material or a material with a density of shallow traps will suffice. The equation for SCLC is ${ }^{17}$

$$
J=\frac{9 \mu \epsilon_{0} \epsilon_{r}}{8 d} E^{2},
$$

where $\mu$ is the carrier mobility, $\epsilon_{r}$ is the relative permittivity, $\epsilon_{0}$ is the permittivity of free space, and $d$ is the film thickness. It can be seen that for different thicknesses $J / d$ will be proportional to $V / d^{2}$ with a slope of 2 when plotted on log$\log$ axes. This is shown in Fig. 2(c) where it can be seen that the curves appear to coincide in the high-field region. The slope of this section is 2.0 which is a remarkable result when compared to the theoretical value. As has been previously stated the low-field conduction follows a linear dependence with voltage and this would explain the deviation from the fit to SCLC at lower electric fields.

Assuming a value of $\epsilon_{r}$ to be 2.6 (the square of the refractive index), by fitting to the equation, a mobility value of $1.2 \times 10^{-10} \mathrm{~cm}^{2} \mathrm{~V}^{-1} \mathrm{~s}^{-1}$ is derived, which is extremely low when compared to other semiconducting films, for example $a-\mathrm{Si}: \mathrm{H}$ which possesses a room temperature mobility of the order of $1 \mathrm{~cm}^{2} \mathrm{~V}^{-1} \mathrm{~s}^{-1}$. ${ }^{16}$ The mobility of $t a-\mathrm{C}$ has been derived by Clough et al. ${ }^{25}$ from measurements of a field-effect transistor to be in the order of $\sim 10^{-6} \mathrm{~cm}^{2} \mathrm{~V}^{-1} \mathrm{~s}^{-1}$. However, the resistivity of $t a-\mathrm{C}$ is commonly in the order of $10^{7} \Omega \mathrm{cm}^{-1}$ (Ref. 26) which is far lower than the measured value for PAC films in this study, $\sim 10^{15} \Omega \mathrm{cm}^{-1}$. The $J-V$ properties of PAC coupled with its low mobility indicate either a trap-free case where the density of transport sites is low, or the case where the mobility is strongly reduced by trapping, resulting in a reduction in the free charge to trapped charge ratio. The low paramagnetic defect density of PAC of $\sim 10^{17}$ spins $\mathrm{cm}^{-3}$ (Ref. 9) would indicate the former in this case. The low mobility of carriers in PAC films therefore results in the high resistivity values which are observed.

The negligible value of $E_{\text {act }}$ for the earthed electrode grown films below $100^{\circ} \mathrm{C}$, as shown in Fig. 3, suggests that any strongly thermally activated mechanism such as extended state or band tail conduction is ruled out for PAC films at room temperature, and therefore conduction at these temperatures and low electric fields is dominated by hopping. It has been suggested ${ }^{21}$ that in the absence of an activation energy, the majority of carriers would be condensed in traps or donor sites. If the centers are closely spaced, the carriers may tunnel between nearest neighbor sites, which is known as tunnel hopping. Due to the lack of any clear temperature dependence, and also the fact that due to the high resistivity of the films any variation is within the experimental error of the measurement apparatus, it is not possible in our case to ascertain whether this type of hopping dominates or another mechanism.

At higher temperatures the value of $E_{\text {act }}$ has been shown from Fig. 3 to be approximately $0.7 \mathrm{eV}$. The most likely mechanism is the transport of carriers excited to localized states at the band edges, otherwise known as band-tail conduction, as one would expect a change from hopping to band-tail conduction as the temperature was increased, rather than a transition directly to transport in the extended states. The value of band gap obtained from the Tauc method is approximately $2.5 \mathrm{eV}$. Therefore, assuming that the Tauc method describes the energy gap between the band edges assuming that they are parabolic, the Fermi level is not at midgap but at $0.7 \mathrm{eV}$ from one of the band edges. Previous studies have shown that intrinsically grown $a$-C is $p$-type $e^{27}$ and an explanation for this is that the dangling bond defect in carbon has a lowered energy leading to $p$-type conductivity. In this case, it is therefore more likely that the $0.7 \mathrm{eV}$ activation energy represents the energy difference between the Fermi level and the valence rather than the conduction band edge.

The $J$ versus $E$ characteristics as a function of ion dose are shown in Fig. 4. Apart from the curve at $2 \times 10^{15} \mathrm{~cm}^{-2}$ the characteristics are symmetric and this indicates bulklimited conduction. The $J / E$ at the lowest doses are consistent with space-charge-limited currents in the bulk of the film, ${ }^{17}$ with the high field regime showing an approximately parabolic $J$ versus $E$ relationship, as described earlier. The large increase in conductivity with increasing boron dose is attributed to a number of factors related to restructuring and defect creation during ion bombardment. It is known that ion damage increases the amount of disorder in the amorphous silicon alloys which increases the strength of the band tails and reduces the effective band gap. ${ }^{28}$ A similar effect is expected in amorphous carbon, but in addition any change in the proportion and distribution of $s p^{2}$ and $s p^{3}$ bonding dur- 
ing bond breaking and reformation will manifest itself in a change in the optical band gap. Earlier it has also been shown that there is an onset for $s p^{2}$ reordering, which is in the range of $10^{14}-10^{15} \mathrm{~cm}^{-2}$.

For implantations at a dose of $6 \times 10^{14} \mathrm{~cm}^{-2}$ and below, the $J / E$ characteristic remains parabolic which suggests SCLC, even though the conductivity has increased over four orders of magnitude. Applying Eq. (4) yields a mobility value of approximately $2 \times 10^{-9} \mathrm{~cm}^{2} \mathrm{~V}^{-1} \mathrm{~s}^{-1}$ for the film implanted at a dose of $6 \times 10^{14} \mathrm{~cm}^{-2}$. This is an increase of over one order of magnitude when compared to the unimplanted case. Therefore, the increase in current magnitude up to a dose of $6 \times 10^{14} \mathrm{~cm}^{-2}$ may be attributed to an increase in low field conductivity, coupled with an increase in mobility which manifests itself at higher fields.

We have previously shown a very strong dose dependence of the band gap and spin density as determined using electron paramagnetic resonance, with the band gap falling from its initial value of 2.6 to $0.4 \mathrm{eV}$ at the highest dose, and the spin density increasing from an initial value of $\sim 10^{17}$ to a final value of $\sim 10^{20} \mathrm{~cm}^{-3}$. ${ }^{11}$ Therefore, as the ion dose increases, there is a narrowing of the band gap, an increase in defect density, and an increase in the doping concentration. All these features promote the transport of carriers via the Frenkel-Poole effect. Indeed, at the highest dose, the $J / E$ characteristics are well described by the hopping of carriers between defect states in the bulk of the film, as a straight line $\log J / E$ versus $E^{1 / 2}$ relationship is observed, which is consistent with Frenkel-Poole conduction. ${ }^{29}$ Since this film is heavily doped, it seems reasonable to assume that the current is due to transport between charged defect centers.

The samples implanted at a dose of $2 \times 10^{15} \mathrm{~cm}^{-2}$ with thicknesses of 80 and $104 \mathrm{~nm}$ have been shown in Fig. 5. Here, the Tauc optical gap is $1.3 \mathrm{eV}$ and Frenkel-Poole conduction appears not to apply, as the calculation performed previously reveals a permittivity value of about 50, far higher than that expected. The presence of hysteresis at the intermediate dose indicates that we have a trapping effect. During the first positive sweep, electrons would be injected from the bottom contact and holes from the top contact. One possible explanation is that the trapping of holes may enhance the electron current. If so, the number of trapped holes will depend on the magnitude of the positive voltage applied to the top current and how long the voltage is applied. Changing any one of these parameters was found to change the size of the loop. On reducing the positive voltage back to zero, holes would be expected to remain trapped so the electron current would also be higher on the way down than on the way up, leading to a loop in the characteristic, as is observed. Similarly, for the $80 \mathrm{~nm}$ thick sample the negative characteristic shows loop behavior. This would also be consistent with the above hypothesis, though this time the holes will be trapped from the back contact.

Further evidence for a current enhancement due to a trapping process is shown in the current versus time measurement as shown in Fig. 6. In this case, it has been observed that for low applied voltages the measured current density decreases with time. This is consistent with a capacitive effect which, when the curve is fitted to an exponential decay, yields a time constant value of approximately $1 \mathrm{~s}$. Applying $C=A \epsilon_{0} \epsilon_{r} / d$ and $R=\rho d / A$, the theoretical time constant for this device is $\rho \epsilon_{0} \epsilon_{r}$ which has been calculated to be $0.2 \mathrm{~s}$. However, at higher measurement voltages, the time constant increases until a voltage of $3 \mathrm{~V}$ at which the current increases rather than decreases with time. This may be explained if the trapping of carriers causes an increases in current magnitude, and the process is less significant at low measurement voltages where it merely counteracts the capacitive decay of the current. At higher measurement voltages, as the voltage is applied, more carriers are trapped which causes a further increase in current over time.

Furthermore, as shown in Fig. 7, below a measurement temperature of $80^{\circ} \mathrm{C}$, as the measurement temperature is increased the current magnitude decreases. Should the current be enhanced by trapping, an increase in temperature is likely to depopulate these traps via a thermionic emission mechanism. It has been mentioned that above a measurement temperature of $80^{\circ} \mathrm{C}$, the hysteresis is absent and the current at higher temperatures then increases with temperature. Above this temperature, therefore, the measurements are consistent with the model assuming that there are no longer any trapped carriers which cause the current enhancement.

The relationship between the trapping of carriers and the presence of loops in the $J / E$ characteristic is supported by the data shown above for the $104 \mathrm{~nm}$ thick film. In this case, the boron ions were unable to penetrate to the bottom part of the film when implanted with the same energy and dose as above. In the negative voltage segment, the current enhancement and hysteresis are missing, and therefore the device shows rectification of approximately one order of magnitude. This may be interpreted as an absence of hole trapping from the bottom contact into the unbombarded part of the $a-\mathrm{C}: \mathrm{H}$ film. The reverse current is therefore unmodified by the presence of holes. The fact that the hysteresis occurs for the positive but not the negative sweep suggests that it is the movement of holes through the material from the positively biased contact that is important rather than the presence of boron atoms that modify the space charge. If the latter was the case then we would have hysteresis in both directions for the partially implanted layer. These results also indicate the holes are the trapped carriers rather than the electrons, due to the polarity of the hysteresis.

Our model for the process described for the above film assumes that conduction remains space-charge limited. Therefore, hole trapping will have the effect of reducing the magnitude of the negative space charge within the film, and will therefore increase the magnitude of the electron current. This will only occur if there were centers to trap holes in the material, for example due to ion-implantation damage, and there was a significant electric field, in order for sufficient hole injection to occur. Such a model is shown in Fig. 8. If there are no centers that trap holes, then the space charge would remain unperturbed and hence no current increase and hysteresis would be observed. This appears to be the case in the $104 \mathrm{~nm}$ thick film, where the absence of current enhancement in the negative voltage sweep suggests insufficient centers to allow hole trapping from the bottom contact. 


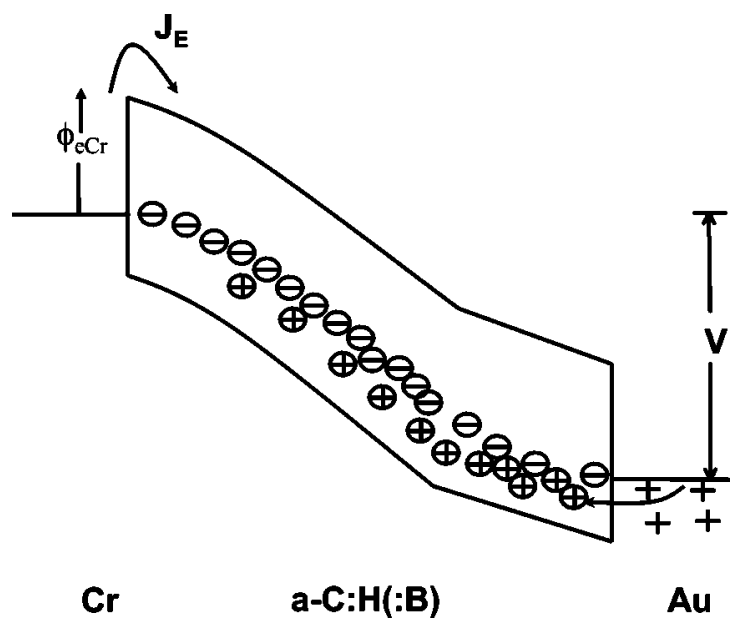

FIG. 8. Proposed band diagram, showing the modification of trapped space charge due to hole injection and trapping from the positively biased contact.

\section{CONCLUSION}

Polymeric amorphous carbon films have been shown to exhibit low-field room-temperature conduction most closely resembling hopping conduction with a very low activation energy. At higher temperatures, the variation of conductivity with temperature suggests that the Fermi level is $0.7 \mathrm{eV}$ from the valence band edge. Conduction at higher electric fields most closely resembles trap-free space-charge-limited current. This is expected, due to the wide band gap and low defect density of the material. We observe that the implantation of boron into this material changes the conductivity of a MSM diode by over eight orders of magnitude. For low and high doses the $J / E$ characteristics are symmetrical about zero voltage which indicates that the current is determined by bulk effects in the film, and the current remains spacecharge limited for the lowest boron doses. An increase in the boron dose increases the amount of damage, changes the balance of the $s p^{2} / s p^{3}$ content, and therefore reduces the band gap, and there is a corresponding increase in the conductivity via Frenkel-Poole type conduction.

Bipolar effects have been observed at an intermediate ion dose, which manifest themselves in the current enhancement with applied electric field and hysteresis in the $J / E$ characteristic. $J / E$ versus temperature and current versus time measurements have suggested that the trapping of carriers results in an enhancement of current magnitude which is both voltage and temperature dependent. The dependence on the back contact and film thickness suggests that holes rather than electrons are trapped which causes a modification of the electron current. A model based on a current enhancement due to the reduction in space charge within the film has been postulated. This phenomenon has not been previously observed and may be of significance for understanding how trapping processes may be utilized to modify the injection and transport processes within thin film materials.

\section{ACKNOWLEDGMENTS}

The authors would like to thank A. Nejim and R. M. Gwilliam at the Nodus Laboratory, University of Surrey, for the performance of the ion implantation. R.U.A.K. also thanks the U. K. Engineering and Physics Research Council for financial support.

${ }^{1}$ P. Koidl, C. Wild, B. Dischler, J. Wagner, and M. Ramsteiner, Mater. Sci. Forum 52-53, 41 (1989).

${ }^{2}$ B. S. Satyanarayana, A. Hart, W. I. Milne, and J. Robertson, Appl. Phys. Lett. 71, 1430 (1997).

${ }^{3} \mathrm{H}$. Hofsäss, in Proceedings, 1st International Specialist Meeting on Amorphous Carbon, edited by S. R. P. Silva, J. Robertson, W. I. Milne, and G. A. J. Amaratunga (World Scientific, Cambridge, England, 1997), pp. 296310.

${ }^{4}$ E. G. Gerstner and D. R. McKenzie, J. Appl. Phys. 84, 5647 (1998).

${ }^{5}$ R. C. Barklie, M. Collins, and S. R. P. Silva, Phys. Rev. B 61, 3546 (2000).

${ }^{6}$ E. Ohta, Y. Kimura, H. Kondo, M. Takahashi, K. Kameyama, K. Yamada, and I. Fujimura, 22nd International Conference of Solid State Devices and Materials (Develpements of International Academic, Sendai, Japan, 1990), p. 589.

${ }^{7}$ S. Egret, J. Robertson, W. I. Milne, and F. J. Clough, Diamond Relat. Mater. 6, 879 (1997).

${ }^{8}$ S. Paul and F. J. Clough, Diamond Relat. Mater. 7, 1734 (1998).

${ }^{9}$ M. Collins, R. C. Barklie, J. V. Anguita, J. D. Carey, and S. R. P. Silva, Diamond Relat. Mater. 9, 781 (2000).

${ }^{10}$ R. D. Forrest, A. P. Burden, S. R. P. Silva, L. K. Cheah, and X. Shi, Appl. Phys. Lett. 73, 3784 (1998)

${ }^{11}$ R. U. A. Khan, J. D. Carey, S. R. P. Silva, B. J. Jones, and R. C. Barklie, Phys. Rev. B 63, 121201 (2001).

${ }^{12}$ M. E. Adel, R. Kalish, and S. Prawer, J. Appl. Phys. 62, 4096 (1987).

${ }^{13}$ D. G. McCulloch, E. G. Gerstner, D. R. McKenzie, S. Prawer, and R. Kalish, Phys. Rev. B 52, 850 (1995).

${ }^{14}$ G. L. Doll, J. P. Heremans, T. A. Perry, and J. V. Mantese, J. Mater. Res. 9, 85 (1994).

${ }^{15}$ N. F. Mott and E. A. Davis, Electronic Processes in Non-Crystalline Materials (Oxford University Press, New York, 1971).

${ }^{16}$ R. A. Street, Hydrogenated Amorphous Silicon (Cambridge University Press, Cambridge, England 1991).

${ }^{17}$ A. Rose, Phys. Rev. 97, 1538 (1955).

${ }^{18}$ J. Frenkel, Phys. Rev. 54, 647 (1938).

${ }^{19}$ E. H. Rhoderick and R. H. Williams, Metal-Semiconductor Contacts (Clarendon, Oxford, U.K., 1988).

${ }^{20}$ P. Mark and T. E. Hartman, J. Appl. Phys. 39, 2163 (1968).

${ }^{21}$ J. G. Simmons, Phys. Rev. 155, 657 (1967).

${ }^{22}$ R. M. Hill, Philos. Mag. 23, 59 (1971).

${ }^{23}$ S. R. P. Silva and G. A. J. Amaratunga, Thin Solid Films 253, 146 (1994).

${ }^{24}$ V. S. Veerasamy, G. A. J. Amaratunga, C. A. Davis, W. I. Milne, P. Hewitt, and M. Weiler, Solid-State Electron. 37, 319 (1994).

${ }^{25}$ F. J. Clough, W. I. Milne, B. Kleinsorge, J. Robertson, G. A. J. Amaratunga, and B. N. Roy, Electron. Lett. 32, 498 (1996).

${ }^{26}$ G. A. J. Amaratunga, D. E. Segal, and D. R. McKenzie, Appl. Phys. Lett. 59, 69 (1991).

${ }^{27}$ S. R. P. Silva, J. Robertson, G. A. J. Amaratunga, B. Rafferty, L. M. Brown, J. Schwan, D. F. Franceschini, and G. Mariotto, J. Appl. Phys. 81, 2626 (1997).

${ }^{28}$ R. A. C. M. M. van Swaaij, A. D. Annis, B. J. Sealy, and J. M. Shannon, J. Appl. Phys. 82, 4800 (1997).

${ }^{29}$ J. G. Simmons, J. Phys. D 4, 613 (1971). 